\title{
SIMULTANEOUS MULTIWAVELENGTH OBSERVATIONS OF MARKARIAN 421 DURING OUTBURST
}

V. A. Acciari ${ }^{1}$, E. Aliu ${ }^{2}$, T. Aune ${ }^{3}$, M. Beilicke ${ }^{4}$, W. Benbow ${ }^{1}$, M. BötTcher ${ }^{5}$, S. M. Bradbury ${ }^{6}$, J. H. Buckley ${ }^{4}$,

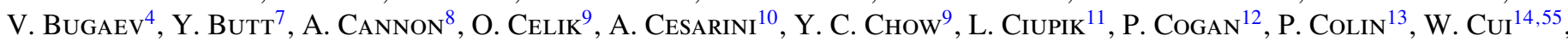
R. Dickherber ${ }^{4}$, C. Duke ${ }^{15}$, A. D. Falcone ${ }^{16}$, S. J. Fegan ${ }^{9}$, J. P. Finley ${ }^{14}$, G. Finnegan ${ }^{13}$, P. Fortin ${ }^{17}$, L. Fortson ${ }^{11}$,

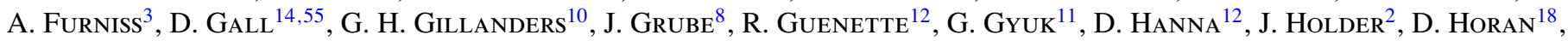
C. M. Hui ${ }^{13}$, T. B. HumenskY ${ }^{19}$, P. KAaret ${ }^{20}$, N. Karlsson ${ }^{11}$, M. KertZman ${ }^{21}$, D. KIeda ${ }^{13}$, J. Kildea ${ }^{1}$, A. Konopelko ${ }^{22}$,

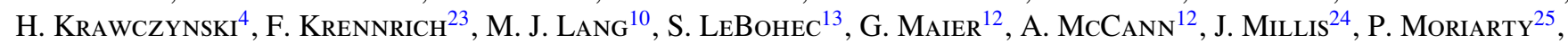
R. A. Ong ${ }^{9}$, A. N. Otte ${ }^{3}$, D. Pandel ${ }^{20}$, J. S. Perkins ${ }^{1}$, A. Pichel ${ }^{26}$, M. Pohl ${ }^{23}$, J. QuinN ${ }^{8}$, K. Ragan ${ }^{12}$, L. C. Reyes ${ }^{27}$, P. T. Reynolds ${ }^{28}$, E. Roache ${ }^{1}$, H. J. Rose ${ }^{6}$, M. Schroedter ${ }^{23}$, G. H. Sembroski ${ }^{14}$, A. W. Smith ${ }^{29}$, D. Steele ${ }^{11}$, S. P. Swordy ${ }^{19}$, M. Theiling ${ }^{1}$, J. A. Toner ${ }^{10}$, A. Varlotta ${ }^{14}$, S. Vincent ${ }^{13}$, S. P. Wakely ${ }^{19}$, J. E. Ward ${ }^{8}$, T. C. Weekes ${ }^{1}$, A. Weinstein ${ }^{9}$,

\section{T. Weisgarber ${ }^{19}$, D. A. Williams ${ }^{3}$, S. WisseL ${ }^{19}$, and B. ZitZer ${ }^{14}$}

(The VERITAS COLlaboration)

I. de la Calle Perez ${ }^{30}$, A. Ibarra ${ }^{30}$, and P. Rodriguez H. Anderhub ${ }^{31}$,

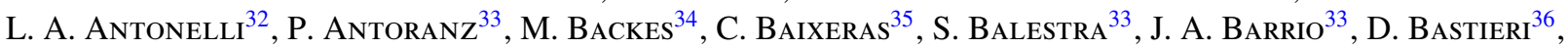
J. Becerra GonZÁlez ${ }^{37}$, J. K. BeCKer ${ }^{34}$, W. Bednarek ${ }^{38}$, K. Berger $^{38}$, E. Bernardini ${ }^{39}$, A. Biland ${ }^{31}$, R. K. Bock ${ }^{40,36}$, G. Bonnoli $^{41}$, P. Bordas ${ }^{42}$, D. Borla Tridon ${ }^{40}$, V. Bosch-Ramon ${ }^{42}$, D. Bose ${ }^{33}$, I. Braun ${ }^{31}$, T. Bretz ${ }^{43}$, I. Britvitch ${ }^{31}$, M. Camara ${ }^{33}$, E. Carmona ${ }^{40}$, A. Carosi ${ }^{32}$, S. Commichau ${ }^{31}$, J. L. Contreras ${ }^{33}$, J. Cortina ${ }^{44}$, M. T. Costado ${ }^{37,45}$, S. Covino $^{32}$, V. Curter ${ }^{34}$, F. Dazzi ${ }^{46,56}$, A. De Angelis ${ }^{46}$, E. De Cea Del Pozo ${ }^{47}$, C. Delgado Mendez ${ }^{37}$, R. De los Reyes ${ }^{33}$, B. De Lotto $^{46}$, M. De Maria ${ }^{46}$, F. De Sabata ${ }^{46}$, A. Dominguez ${ }^{48}$, D. Dorner ${ }^{31}$, M. Doro ${ }^{36}$, D. Elsaesser ${ }^{43}$, M. Errando ${ }^{44}$, D. Ferenc ${ }^{49}$, E. Fernández ${ }^{44}$, R. Firpo ${ }^{44}$, M. V. FonseCa ${ }^{33}$, L. Font ${ }^{35}$, N. Galante ${ }^{40}$, R. J. García López ${ }^{37,45}$, M. GarczarczyK ${ }^{44}$, M. Gaug ${ }^{37}$, F. Goebel ${ }^{40,57}$, D. Hadasch ${ }^{35}$, M. Hayashida ${ }^{40}$, A. Herrero ${ }^{37,45}$, D. Hildebrand ${ }^{31}$, D. HöHNe-MöNCH ${ }^{43}$, J. $\mathrm{Hose}^{40}$, C. C. $\mathrm{Hsu}^{40}$, T. Jogler ${ }^{40}$, D. Kranich ${ }^{31}$, A. La Barbera ${ }^{32}$, A. Laille ${ }^{49}$, E. LeOnardo ${ }^{41}$,

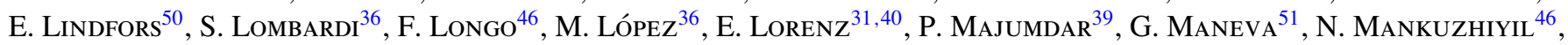
K. Mannheim ${ }^{43}$, L. Maraschi ${ }^{32}$, M. Mariotti ${ }^{36}$, M. Martínez ${ }^{44}$, D. Mazin ${ }^{44}$, M. Meucci ${ }^{41}$, J. M. Miranda ${ }^{33}$, R. Mirzoyan ${ }^{40}$, H. Miyamoto ${ }^{40}$, J. Moldón ${ }^{42}$, M. Moles ${ }^{48}$, A. Moralejo ${ }^{44}$, D. Nieto ${ }^{33}$, K. Nilsson ${ }^{50}$, J. Ninkovic ${ }^{40}$, R. Orito ${ }^{40}$, I. Oya ${ }^{33}$, R. Padeletti ${ }^{41}$, J. M. Paredes ${ }^{42}$, M. Pasanen ${ }^{50}$, D. Pascoli ${ }^{36}$, F. Pauss ${ }^{31}$, R. G. Pegna ${ }^{41}$, M. A. Perez-Torres ${ }^{48}$, M. Persic ${ }^{46,52}$, L. Peruzzo ${ }^{36}$, F. Prada ${ }^{48}$, E. Prandini ${ }^{36}$, N. Puchades ${ }^{44}$, I. Reichardi ${ }^{44}$, W. Rhode ${ }^{34}$, M. Ribó ${ }^{42}$, J. Rico ${ }^{53,44}$, M. Rissi ${ }^{31}$,

A. Robert ${ }^{35}$, S. RüGamer ${ }^{43}$, A. SAgGion ${ }^{36}$, T. Y. Saito ${ }^{40}$, M. Salvati ${ }^{32}$, M. SAnchez-Conde ${ }^{48}$, K. Satalecka ${ }^{39}$,

V. Scalzotto ${ }^{36}$, V. Scapin ${ }^{46}$, T. Schweizer ${ }^{40}$, M. Shayduk ${ }^{40}$, S. N. Shore ${ }^{54}$, N. Sidro ${ }^{44}$, A. Sierpowska-Bartosik ${ }^{47}$,

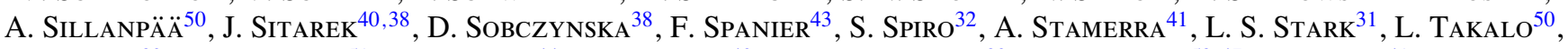

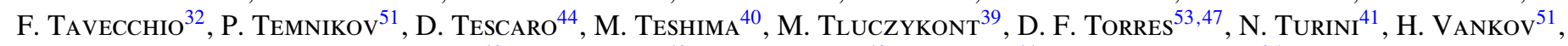
R. M. WAGNER ${ }^{40}$, V. ZABALZA ${ }^{42}$, F. ZANDANEL ${ }^{48}$, R. ZANIN ${ }^{44}$, AND J. ZAPATERo ${ }^{35}$

(The MAGIC COLlaboration)

${ }^{1}$ Fred Lawrence Whipple Observatory, Harvard-Smithsonian Center for Astrophysics, Amado, AZ 85645, USA

2 Department of Physics and Astronomy and the Bartol Research Institute, University of Delaware, Newark, DE 19716, USA

${ }^{3}$ Santa Cruz Institute for Particle Physics and Department of Physics, University of California, Santa Cruz, CA 95064, USA

${ }^{4}$ Department of Physics, Washington University, St. Louis, MO 63130, USA

${ }^{5}$ Astrophysical Institute, Department of Physics and Astronomy, Ohio University, Athens, OH 45701, USA

${ }^{6}$ School of Physics and Astronomy, University of Leeds, Leeds, LS2 9JT, UK

${ }^{7}$ Harvard-Smithsonian Center for Astrophysics, 60 Garden Street, Cambridge, MA 02138, USA

${ }^{8}$ School of Physics, University College Dublin, Belfield, Dublin 4, Republic of Ireland

${ }^{9}$ Department of Physics and Astronomy, University of California, Los Angeles, CA 90095, USA

${ }^{10}$ School of Physics, National University of Ireland, Galway, Republic of Ireland

${ }^{11}$ Astronomy Department, Adler Planetarium and Astronomy Museum, Chicago, IL 60605, USA

${ }_{12}^{12}$ Physics Department, McGill University, Montreal, QC H3A 2T8, Canada

${ }^{13}$ Physics Department, University of Utah, Salt Lake City, UT 84112, USA

${ }^{14}$ Department of Physics, Purdue University, West Lafayette, IN 47907, USA; dgall@ physics.purdue.edu, cui@physics.purdue.edu

${ }^{15}$ Department of Physics, Grinnell College, Grinnell, IA 50112-1690, USA

${ }^{16}$ Department of Astronomy \& Astrophysics, Pennsylvania State University, University Park, PA 16802, USA

${ }^{17}$ Department of Physics and Astronomy, Barnard College, Columbia University, NY 10027, USA

${ }^{18}$ Laboratoire Leprince-Ringuet, Ecole Polytechnique, CNRS/IN2P3, F-91128 Palaiseau, France

${ }^{19}$ Enrico Fermi Institute, University of Chicago, Chicago, IL 60637, USA

${ }^{20}$ Department of Physics and Astronomy, University of Iowa, Van Allen Hall, Iowa City, IA 52242, USA

${ }^{21}$ Department of Physics and Astronomy, DePauw University, Greencastle, IN 46135-0037, USA

${ }^{22}$ Department of Physics, Pittsburg State University, 1701 South Broadway, Pittsburg, KS 66762, USA

${ }^{23}$ Department of Physics and Astronomy, Iowa State University, Ames, IA 50011, USA

${ }^{24}$ Department of Physics, Anderson University, 1100 East 5th Street, Anderson, IN 46012, USA

${ }^{25}$ Department of Life and Physical Sciences, Galway-Mayo Institute of Technology, Dublin Road, Galway, Republic of Ireland

${ }^{26}$ Instituto de Astronomia y Fisica del Espacio, Casilla de Correo 67 - Sucursal 28, (C1428ZAA) Ciudad Autnoma de Buenos Aires, Argentina

${ }^{27}$ Kavli Institute for Cosmological Physics, University of Chicago, Chicago, IL 60637, USA

${ }^{28}$ Department of Applied Physics and Instrumentation, Cork Institute of Technology, Bishopstown, Cork, Republic of Ireland ${ }^{29}$ Argonne National Laboratory, 9700 S. Cass Avenue, Argonne, IL 60439, USA

${ }^{30}$ XMM-Newton-SOC, European Space Astronomy Centre, ESA, 28691 Villanueva de la Cañada (Madrid), Spain 


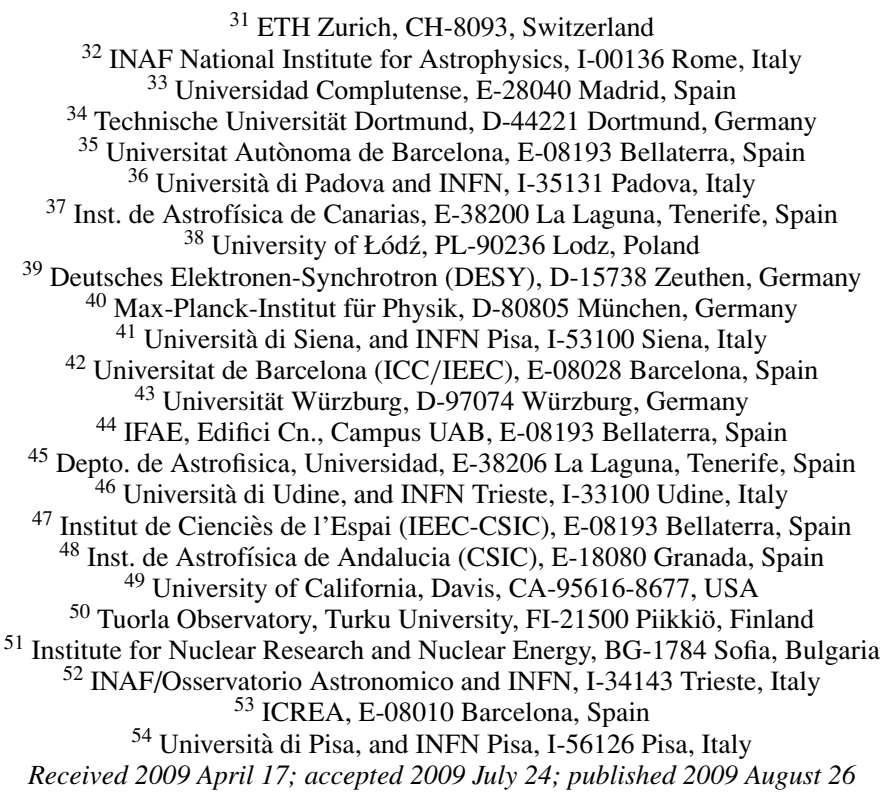

ABSTRACT

We report on the results of two coordinated multiwavelength campaigns that focused on the blazar Markarian 421 during its 2006 and 2008 outbursts. These campaigns obtained UV and X-ray data using the XMM-Newton satellite, while the gamma-ray data were obtained utilizing three imaging atmospheric Cerenkov telescopes, the Whipple $10 \mathrm{~m}$ telescope and VERITAS, both based in Arizona, as well as the MAGIC telescope, based on La Palma in the Canary Islands. The coordinated effort between the gamma-ray groups allowed for truly simultaneous data in UV/X-ray/gamma-ray wavelengths during a significant portion of the XMM-Newton observations. This simultaneous coverage allowed for a reliable search for correlations between UV, X-ray, and gamma-ray variability over the course of the observations. Investigations of spectral hysteresis and modeling of the spectral energy distributions are also presented.

Key words: BL Lacertae objects: individual (Markarian 421) - galaxies: active - gamma rays: observations radiation mechanisms: non-thermal - X-rays: galaxies

\section{INTRODUCTION}

Blazars, a subclass of active galactic nuclei (AGNs), are some of the most intriguing sources in the high-energy sky. Their rapid variability and the nonthermal nature of their emission, presenting a continuum across nearly the entire electromagnetic spectrum, imply that the observed photons originated within highly relativistic jets oriented very close to the observer's line of sight (Urry \& Padovani 1995). Therefore, blazars are excellent laboratories for studying the physical processes within the jets of AGNs. They were among the first very high energy (VHE; $E>100 \mathrm{GeV}$ ) sources to be detected and today there are 25 known VHE blazars, including flat spectrum radio quasars and BL Lac objects.

Various models have been proposed to account for the broadband spectral energy distributions (SEDs) observed in VHE blazars, which typically display a characteristic double peak when plotted as $v F_{v}$ against $\nu$, with peaks occurring at $\mathrm{keV}$ and $\mathrm{TeV}$ energies. The models are generally divided into two classes: leptonic and hadronic. Both leptonic and hadronic models attribute the peak at $\mathrm{keV}$ energies to synchrotron radiation from relativistic electrons (and positrons) within the

\footnotetext{
55 Authors to whom any correspondence should be addressed.

56 Supported by INFN Padova.

${ }^{57}$ Deceased.
}

jet, but they differ on the origin of the $\mathrm{TeV}$ peak. The leptonic models advocate the inverse Compton scattering mechanism, utilizing synchrotron self-Compton (SSC) interactions and/or inverse Compton interactions with an external photon field, to explain the VHE emission (e.g., Marscher \& Gear 1985; Maraschi et al. 1992; Dermer et al. 1992; Sikora et al. 1994). The hadronic models, however, account for the VHE emission by $\pi^{0}$ or charged pion decay with subsequent synchrotron and/or Compton emission from decay products, or synchrotron radiation from ultrarelativistic protons (e.g., Mannheim 1993; Aharonian 2000; Pohl \& Schlickeiser 2000).

Observationally, blazars are known to undergo both major outbursts on long time scales and rapid flares on short time scales, most prominently at $\mathrm{keV}$ and $\mathrm{TeV}$ energies. During some outbursts, both of the SED peaks have been observed to shift toward higher energies in a generally correlated manner (e.g., Błażejowski et al. 2005). The correlation of the variabilities at $\mathrm{keV}$ and $\mathrm{TeV}$ energies (or lack thereof) during such outbursts has aided in refining the emission models. In addition, rapid, sub-hour flaring is interesting as it provides direct constraints on the size of the emission region. These rapid flares also present an observational challenge to multiwavelength studies, as truly simultaneous data must be used in order to develop a reliable characterization of the broadband behavior of these objects.

Markarian 421 (Mrk421; 1101+384), at a redshift of $z=$ 0.031 , was the first blazar, as well as the first extragalactic 
source, to be detected at $\mathrm{TeV}$ energies (Punch et al. 1992) and has since remained one of the most active VHE blazars. Its SED has peaks at $\mathrm{keV}$ and $\mathrm{TeV}$ energies, and it has been known to demonstrate rapid, sub-hour, flaring behavior at these energies during the course of an outburst (e.g., Cui 2004; Gaidos et al. 1996) indicating very compact emission regions. Many extensive multiwavelength campaigns studying Markarian 421 have been undertaken at $\mathrm{keV}$ and $\mathrm{TeV}$ energies during outbursts, but the degree of simultaneity of the multiwavelength coverage varies and is often not adequate to account for the most rapid variability of the source. Due to these considerations, one must exercise caution in the interpretation of some of the results from these campaigns.

In this work, we report on results from a target-of-opportunity (ToO) program on VHE blazars that makes use of a unique combination of capabilities provided by the XMM-Newton satellite and several ground-based imaging atmospheric Cerenkov Telescopes (IACTs) to obtain truly simultaneous coverage in the optical/UV, X-ray, and VHE bands. Unlike other satellites frequently used for multiwavelength campaigns, XMM-Newton's highly elliptical orbit allows for long observations that are not frequently interrupted by earth occultation. In addition to its $\mathrm{X}$-ray instruments, XMM-Newton carries an optical/UV telescope co-aligned with the X-ray telescopes and can thus provide simultaneous coverage in the optical/UV band. While our primary emphasis is on the X-ray and VHE bands, the optical/UV coverage helps to constrain the overall SED shape.

\section{OBSERVATIONS AND DATA REDUCTION}

Our ToO program was first triggered in April 2006 by a major outburst from Markarian 421 as detected by regular monitoring of the VHE band by the Whipple $10 \mathrm{~m}$ telescope. Because of XMM-Newton visibility constraints, the coordinated multiwavelength observations did not take place until after the peak of the outburst as indicated by the overall monitoring campaign. The program was triggered again in May 2008 by another major outburst from Markarian 421 detected in the VHE band. We once again captured only the decaying portion of the outburst. However, taken together, the two campaigns have produced a significant amount of simultaneous optical/ UV, X-ray, and VHE data on the source.

\subsection{XMM-Newton Observations}

The X-ray and optical/UV observations were taken by the XMM-Newton satellite's EPIC-pn (EPN) detector (Strüder et al. 2001 ), covering a spectral range of approximately $0.5-10 \mathrm{keV}$, and the Optical Monitor (OM; Mason et al. 2001), capable of covering the range between 170 and $650 \mathrm{~nm}(7.3 \mathrm{eV}$ and $1.9 \mathrm{eV}$ ). The metal oxide semiconductor (MOS) detectors were also operated during some of the observations, but the data were not used here. During both observations, the EPN was operated in fast timing mode to minimize photon pileup at the expense of imaging capability along the direction of event readout. The thin optical filter was in place for both observations. The 2006 and 2008 observations produced EPN exposures of approximately $42 \mathrm{ks}$ and $43 \mathrm{ks}$, respectively. Due to the brightness of the source, during the first half of the 2008 observations both the EPN and MOS detectors entered into counting mode resulting in a loss of frames. In an attempt to avoid further frame losses, the MOS detectors were shut down to provide more bandwidth for telemetry to the EPN detector. As a result of the detector being in counting mode, the first half of the EPN observation suffers
Table 1

Summary of Markarian 421 XMM-Newton Observations.

\begin{tabular}{ccccc}
\hline \hline Obs. ID & Start Time & $\begin{array}{c}\text { PN Exposure } \\
(\mathrm{ks})\end{array}$ & $\begin{array}{c}\text { Avg. PN Rate } \\
\left(\text { counts s}^{-1}\right)\end{array}$ & $\begin{array}{c}\text { Avg. OM Rate } \\
\left(\text { counts s }^{-1}\right)\end{array}$ \\
\hline 0302180101 & $\begin{array}{c}2006 \text { Apr 29 } \\
\text { 20:44UT }\end{array}$ & 41.9 & $336.30 \pm 0.09$ & $13.5 \pm 0.2$ \\
0502030101 & $\begin{array}{c}2008 \text { May 7 } \\
01: 34 U T\end{array}$ & 43.2 & $411.31 \pm 0.12$ & $35.82 \pm 0.03$ \\
& & & \\
\hline
\end{tabular}

from telemetry gaps which can be accounted for and corrected as explained below.

In parallel with the EPN observations, a series of exposures was taken utilizing the OM. The 2006 and 2008 observations produced 15 and 10 exposures, respectively, with total exposure times of $32.5 \mathrm{ks}$ and $22.0 \mathrm{ks}$. For all exposures, the OM was in imaging mode with the UVM2 filter $(200-300 \mathrm{~nm})$ in place. Table 1 summarizes these observations.

The 2006 EPN data were initially processed using XMMSAS v7.0 (Gabriel et al. 2004). Standard XMM-Newton data analysis procedures were followed to filter and reduce the data, generate the various light curves, extract the source and background spectrum, and generate the RMF and ARF files for subsequent spectral analyses. In summary, the event list was examined to check for periods of soft proton flaring, where background event rates are higher than 0.4 counts $\mathrm{s}^{-1}$ (for events with energies $E>10 \mathrm{keV}$ ). In addition, "bad events" were removed including events which were close to CCD gaps or bad pixels, and only single and double photon events were included (PATTERN $\leqslant$ 4). A one-dimensional histogram of counts was produced to determine the position of the source. Based on the histogram, we chose columns 30-45 (in RAWX) for the source region and columns 1-15 and 57-64 for the background region. These regions were used to extract source and background spectra.

The 2008 EPN data were processed similarly, using the same filtering criteria, but with XMMSAS v8.0. In this case, the source region was determined to be in columns $31-45$ and the background regions were taken to be columns 1-19 and 57-63. It should be noted that a new SAS task, epiclccorr, was used to correct for the effects of deadtime, telemetry saturation, and frame dropouts in producing the light curves from the 2006 and 2008 data.

The OM data were analyzed with the standard SAS task omichain, which produces images and source lists for all OM exposures present in any given observation. The 2006 OM observations presented here are a combination of 15 exposures with $2000 \mathrm{~s}$ and $2500 \mathrm{~s}$ integration times, while the 2008 data comes from 10 exposures each with a duration of $2200 \mathrm{~s}$.

\subsection{VHE Gamma-ray Observations}

Three ground-based IACT facilities were utilized to observe the source in the VHE band during the XMM-Newton observations. These IACTs detect gamma-rays by imaging the flashes of Cerenkov light emitted by gamma-ray induced electromagnetic showers within the atmosphere. The Major Atmospheric Gamma Imaging Cerenkov (MAGIC) telescope provided $14 \mathrm{ks}$ of coverage during the first part of the 2006 XMM-Newton observation, and the Whipple $10 \mathrm{~m}$ telescope provided $12 \mathrm{ks}$ of coverage during the latter part of the 2006 observation. The geographic separation of the experiments allowed for extended, simultaneous coverage during the XMM-Newton observation. During the $2008 X M M-N e w t o n$ observation, only the 
Table 2

Whipple Event Selection Parameters

\begin{tabular}{lc}
\hline \hline \multicolumn{1}{c}{ Parameter } & Values for Cuts \\
\hline Trigger level & $>30$ digital counts \\
Shape cuts & $0.05<$ width $<0.12$ \\
& $0.13<$ length $<0.25$ \\
Muon cut & Length $/$ size $<0.0004 \mathrm{dc}^{-1}$ \\
Distance cut & $0.4<$ distance $<1.0$ \\
Orientation cut & $\alpha<15^{\circ}$ \\
\hline
\end{tabular}

Very Energetic Radiation Imaging Telescope Array System (VERITAS) was used, providing $9 \mathrm{ks}$ of VHE coverage.

\subsubsection{Whipple Data}

The Whipple $10 \mathrm{~m}$ IACT (Kildea et al. 2007) focuses Cerenkov light on a camera composed of 499 photo multiplier tubes (PMTs). The energy threshold for the Whipple $10 \mathrm{~m}$ is near $400 \mathrm{GeV}$. There are two modes used for taking data. During the 2006 observation, $2 \mathrm{hr}$ of data were taken in TRACKING mode, where the source is centered in the camera, and $2 \mathrm{hr}$ of data were taken in ON/OFF mode, where half of the data runs are offset from the source by 30 minutes in right ascension to provide an independent measurement of background events (primarily cosmic rays). The runs each had a duration of 28 minutes, and the total time on source for the Whipple $10 \mathrm{~m}$ telescope was $3 \mathrm{hr}$ on 2006 April 30 from 4:37 UT to 8:05 UT. The observations were taken with a source elevation range of $45^{\circ}-79^{\circ}$, with an average elevation of $64^{\circ}$ and during favorable weather conditions.

Whipple data reduction involves two stages, image cleaning and image parameterization. In the cleaning stage, the data is flat-fielded using a run during which a nitrogen arc lamp is pulsed to illuminate the camera's pixels. Any differences between night-sky background between ON and OFF runs is also accounted for at this stage. In addition, pixels that make up shower images are selected and the remaining background pixels are then removed prior to image parameterization (Punch et al. 1991). During the image parameterization stage, each Cerenkov image in the telescope's camera is characterized by an ellipse, using a moment analysis of the recorded signal amplitudes in each pixel. Each shower is characterized by the major axis (length), minor axis (width), the angle between the major axis of an image's ellipse and a line from the centroid of the ellipse to the source position $(\alpha)$, the length of this line (distance), and the overall signal of the shower (size). These are known as Hillas parameters and they allow for the removal of the primary source of background, cosmic rays, by exploiting the intrinsic differences in the development of hadronic cosmic ray and gamma-ray showers (Hillas 1985). These differences result in different distributions of light at ground level and cuts can be used to select events most likely to be gamma rays. Standard cuts were made on the Hillas parameters, as shown in Table 2, to reject as many background events as possible while still retaining many gamma-ray candidates.

The runs taken in ON/OFF mode obtain a direct measurement of background from the off-source runs, while in TRACKING mode a "tracking ratio" is used to estimate the background. Gamma-ray events should have a small $\alpha$ parameter if the source is centered in the field of view, so events with large values for $\alpha$ can be used to estimate the background if the ratio between background rates at small and large values of $\alpha$ is known in the absence of a source. This "tracking ratio" used here was found by taking observations of blank sky fields with no detected
Table 3

MAGIC Cuts

\begin{tabular}{lc}
\hline \hline \multicolumn{1}{c}{ Parameter } & Values for Cuts \\
\hline Number of shower islands & $<3$ \\
Number of used pixels & $>5$ \\
Leakage & $<0.3$ \\
\hline Gamma/hadron separation cuts & $<0.046$ \\
$\theta^{2}$ & $<0.265 \times\left(1-0.0803 \times(\log 10(\text { size })-5.77)^{2}\right)$ \\
Area & Slope $>($ distance -0.5$) \times 7.2$ \\
Standard time cuts &
\end{tabular}

Note. Leakage parameter is defined as the ratio of the signal in the outer pixels of the camera to the total signal.

VHE sources throughout the 2005-2006 observing season. It is defined as the ratio of the integrated number of events between $\alpha=0^{\circ}-15^{\circ}$ and those between $\alpha=20^{\circ}-65^{\circ}$. Once it is calculated, the tracking ratio is used to estimate background rates for all TRACKING observations.

\subsubsection{MAGIC Data}

MAGIC is the largest single dish Cerenkov telescope in operation (Baixeras et al. 2004; Cortina et al. 2005). A $17 \mathrm{~m}$ tesselated reflector focuses the light from air showers on a camera composed of 576 PMTs. For high elevation angle observations, the MAGIC trigger threshold currently reaches down to 50-60 GeV (Albert et al. 2008).

The measurements reported in this article were conducted from 2006 April 29 21:32 UT to 2006 April 30 00:59 UT at elevation angles spanning $49^{\circ}-81^{\circ}$. MAGIC observed the source employing the so-called wobble mode (Fomin et al. 1994), during which the telescope alternates between tracking two (or more) opposing sky directions, each 0.4 off-source, for 20 minutes each (Anderhub et al. 2009). After removing events not containing sufficient information for further analysis (see Table 3 top), events from accidental triggers, triggers from nearby muons, and data affected by adverse meteorological conditions, $2.8 \mathrm{hr}$ out of the $3.8 \mathrm{hr}$ of data were used for further analysis.

The data were processed using the analysis and reconstruction software package for MAGIC data (Bretz \& Dorner 2008). A description of the different analysis steps can be found in Gaug et al. (2005), Albert et al. (2008), and Bretz (2005). For this analysis, the signal has been extracted using a spline algorithm. After calibration, the shower images were cleaned of background noise by requiring a minimum photoelectron signal in the pixels as well as temporal coincidence with adjacent pixels (time image cleaning; see Anderhub et al. 2009). The recorded events are characterized by several image parameters based on the shower light distribution (among them the previously mentioned Hillas parameters) and on the temporal shower development in the camera plane (slope; see Anderhub et al. 2009). True gamma-ray events coming from the observed source are extracted from the hadronic background by cuts in this image parameter space, using the separation cuts listed at the bottom of Table 3. Events originating from the source are selected by a cut on $\theta^{2}$ (where $\theta$ is the angular distance between the expected source position and the reconstructed gammaray arrival direction). Gamma-ray events are then separated from background events by a size dependent parabolic cut in width $\times$ length $\times \pi$ (Riegel et al 2005).

The Markarian 421 observations presented here are among the first data taken by MAGIC after major hardware updates in April 2006. Due to these changes, a thorough examination 
Table 4

VERITAS Cuts

\begin{tabular}{lc}
\hline \hline \multicolumn{1}{c}{ Parameter } & Values for Cuts \\
\hline Size & $>400$ digital counts \\
Pixels & $\geqslant 5$ \\
Distance & $\leqslant 1.43$ \\
\hline Mean scaled width & $0.05<\mathrm{MSW}<1.16$ \\
Mean scaled length & $0.05<\mathrm{MSL}<1.36$
\end{tabular}

of the data was undertaken to ensure its reliability. Despite the hardware changes, all MAGIC subsystems were working as expected with the exception of an unstable trigger behavior for some PMTs, leading to an early signal arrival time for those affected. A careful study of the systematics in the low-energy region has been conducted, leading to the decision of raising the energy threshold to $250 \mathrm{GeV}$ (Anderhub et al. 2009).

\subsubsection{VERITAS Data}

VERITAS is an array of four $12 \mathrm{~m}$ diameter IACTs each focusing light on a camera consisting of 499 PMTs. Utilizing multiple telescopes provides a stereoscopic view of showers, making it possible to significantly reduce muon events. This substantially improves the low-energy performance of the detector since images produced from nearby muons are otherwise virtually indistinguishable from images produced by low-energy gamma rays. Therefore, the array allows for a lower energy threshold than possible with a single telescope of the same size operating alone. For VERITAS, this threshold is near 275 $\mathrm{GeV}$. In addition, stereoscopic observations facilitate a much improved determination of the core position of showers and thus provide improved energy resolution and background rejection.

During the 2008 observation, VERITAS took data on Markarian 421 while operating in wobble mode, with an offset angle of 0.5 . To provide background estimations using this observation mode, we follow the reflected-region model (Berge, Funk, \& Hinton 2007) where one collection region is placed at the source position and others of equal size are placed at equal offsets from the center of the field of view and used for background measurements. VERITAS observed Markarian 421 for $2.5 \mathrm{hr}$ on 2008 May 7 from 3:59 UT to 6:28 UT using a series of 20 minute runs, with an effective exposure time of 129.7 minutes. The observations were taken as the source elevation ranged from $79^{\circ}$ to $55^{\circ}$. Data quality checks confirmed good weather during the observations.

The VERITAS data from 2008 was analyzed with VERITAS Gamma-ray Analysis Suite (VEGAS), the standard VERITAS analysis package (Cogan et al. 2007). The shower images were first corrected for relative gains and cleaned to remove isolated pixels. The images were then parameterized in a similar manner as for Whipple analysis. Initial quality cuts were made on the images' size, pixel count, and distance. For this analysis, events that survived the initial cuts but only contained data from the two telescopes with the smallest physical separation were removed. This smaller separation distance results in a greater number of muons surviving the initial cuts, and in addition these events can distort the calculation of impact distance due to the relatively small distance between the telescopes. After stereoscopic reconstruction and calculation of each event's impact distance (distance from the shower core to the telescope), the events were parameterized using the quantities mean scaled width (MSW) and mean scaled length (MSL; Konopelko 1995; Daum et al. 1997). These parameters are found by scaling the
Table 5

Gamma-ray Data Run Information

\begin{tabular}{|c|c|c|c|}
\hline Facility/Date & Time & Wobble Direction & Significance $(\sigma)$ \\
\hline \multicolumn{4}{|l|}{ MAGIC } \\
\hline \multirow[t]{7}{*}{2006 Apr 29} & 21:29 UT & $0.40+000$ & 12.0 \\
\hline & 21:49 UT & $0.40+180$ & 9.3 \\
\hline & 22:41 UT & $0.40+180$ & 5.0 \\
\hline & $22: 51 \mathrm{UT}$ & $0.40+000$ & 7.6 \\
\hline & 23:11 UT & $0.40+180$ & 8.4 \\
\hline & 23:31 UT & $0.40+000$ & 8.9 \\
\hline & 23:52 UT & $0.40+180$ & 6.6 \\
\hline \multirow[t]{4}{*}{2006 Apr 30} & $00: 12 \mathrm{UT}$ & $0.40+000$ & 6.0 \\
\hline & 00:32 UT & $0.40+180$ & 5.9 \\
\hline & 00:53 UT & $0.40+000$ & 4.4 \\
\hline & & TOTAL & 23.7 \\
\hline \multicolumn{4}{|l|}{ Whipple } \\
\hline \multirow[t]{7}{*}{2006 Apr 30} & 04:37 UT & TRACKING & 4.9 \\
\hline & 05:05 UT & TRACKING & 5.4 \\
\hline & 05:39 UT & TRACKING & 6.6 \\
\hline & 06:07 UT & TRACKING & 3.7 \\
\hline & 06:37 UT & ON/OFF & 4.8 \\
\hline & 07:37 UT & ON/OFF & 5.3 \\
\hline & & TOTAL & 12.5 \\
\hline \multicolumn{4}{|l|}{ VERITAS } \\
\hline \multirow[t]{8}{*}{2008 May 7} & 03:59 UT & $0.5 \mathrm{~N}$ & 15.5 \\
\hline & 04:21 UT & $0.5 \mathrm{~S}$ & 15.4 \\
\hline & 04:43 UT & $0.5 \mathrm{E}$ & 16.9 \\
\hline & 05:04 UT & $0.5 \mathrm{~W}$ & 13.5 \\
\hline & 05:26 UT & $0.5 \mathrm{~N}$ & 15.2 \\
\hline & 05:47 UT & $0.5 \mathrm{~S}$ & 14.6 \\
\hline & 06:08 UT & $0.5 \mathrm{E}$ & 13.7 \\
\hline & & TOTAL & 39.6 \\
\hline
\end{tabular}

Notes. Some data were removed due to adverse meteorological conditions.

width and length parameters for a given telescope by the average expected values from simulations, given an impact distance and size and then finding the average for all telescopes involved in an event. Cuts on MSW and MSL were made to separate gammaray events from cosmic-ray events. Table 4 details the cuts that are discussed above. A summary of all VHE gamma-ray data is shown in Table 5.

\section{RESULTS}

\subsection{Time Averaged VHE Gamma-ray Spectra}

We followed the procedures from Mohanty et al. (1998) in constructing a spectrum based on the Whipple data. This method depends on separate energy estimates for both on-source and off-source runs, so the runs taken in the TRACKING mode were matched to contemporaneous OFF runs taken at similar elevation angles. The selected matched runs were taken within 2 days of the Markarian 421 observations and at elevation angles within $1^{\circ}$ of the corresponding TRACKING runs. The energy spectrum was fit using a simple power law:

$$
\frac{\mathrm{d} N}{\mathrm{~d} E}=F_{0} \cdot 10^{-11} \cdot\left(\frac{E}{1 \mathrm{TeV}}\right)^{-\alpha} \cdot \frac{\text { photons }}{\mathrm{TeV} \mathrm{cm}^{2} \mathrm{~s}} .
$$

Finding best-fit parameters of $\alpha=2.23 \pm 0.38$ and $F_{0}=$ $2.65 \pm 0.77$ yielding a $\chi^{2} /$ dof of $0.17 / 3(P=98.2 \%)$. Uncertainties are statistical only.

The MAGIC spectrum has been derived on the same data basis as the light curve but a somewhat looser area cut was applied. 


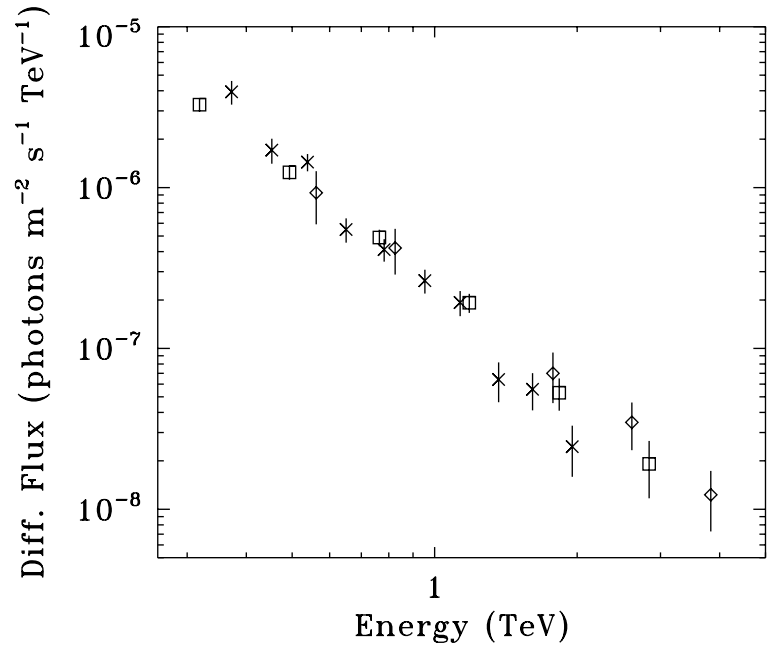

Figure 1. VHE spectral analysis results, showing the data obtained with MAGIC (squares; $\alpha=2.28 \pm 0.09$ and $F_{0}=2.49 \pm 0.17$ ), Whipple (diamonds; $\alpha=2.23 \pm 0.38$ and $F_{0}=2.65 \pm 0.77$ ), and VERITAS (crosses; $\alpha=2.91 \pm 0.13$ and $\left.F_{0}=2.01 \pm 0.15\right)$.

This cut yielded a constant cut efficiency as a function of energy of $90 \%$ for Monte Carlo simulated gamma-ray events, increasing the gamma-ray event statistics at the threshold (Albert et al. 2009). The energies of the gamma events were reconstructed using a random forest regression method (Breiman 2001; Albert et al. 2007c) trained with Monte Carlo events. The MAGIC spectrum was modeled similarly to the Whipple spectrum, finding best fit parameters of $\alpha=2.28 \pm 0.09$ and $F_{0}=$ $2.49 \pm 0.17$, yielding a $\chi^{2} /$ dof of $2.04 / 4(P=72.9 \%)$. All the stated uncertainties for MAGIC are purely statistical. The energy scale is known with an uncertainty of $\pm 16 \%$, the flux normalization within a systematic error of $11 \%$ (not including the energy scale error), and the fitted power-law slope has a systematic uncertainty of \pm 0.2 (Albert et al. 2008).

For the MAGIC analysis, an additional systematic uncertainty is provided to account for possible effects arising from the hardware instability mentioned above. These effects consist of a moderate loss of low-energy showers as well as a minor additional uncertainty in the image parameter calculation for showers of higher energy. The effect on the differential flux level is estimated to be $10 \%$ from $250 \mathrm{GeV}$ to $400 \mathrm{GeV}$ and $3 \%$ for higher energies (Anderhub et al. 2009).

The spectral analysis for the VERITAS data was performed using the VEGAS analysis package (Cogan et al. 2007), with the same model for background estimates as used for the light curve. The spectrum was again fit with a simple power-law model, finding best fit parameters of $\alpha=2.91 \pm 0.13$ and $F_{0}=2.01 \pm 0.15$, yielding a $\chi^{2} /$ dof of $9.66 / 8(P=29.0 \%)$. Again, stated uncertainties are statistical only. The results from the spectral fits for all VHE data can be seen in Figure 1.

\subsection{Spectral Energy Distribution and Modeling}

The X-ray spectrum was initially fit using XSPEC 12 . The data were fit with a power law modified by interstellar absorption, yielding a value for the photon index of $\alpha=2.258 \pm 0.002$ and $2.153 \pm 0.002$ for the data obtained simultaneously with the 2006 MAGIC and Whipple $10 \mathrm{~m}$ observations, respectively. For $\mathrm{X}$-ray data taken during the 2008 VERITAS observations, a photon index of $\alpha=2.519 \pm 0.010$ was found. The hydrogen column density was left as a free parameter for all fits, finding values

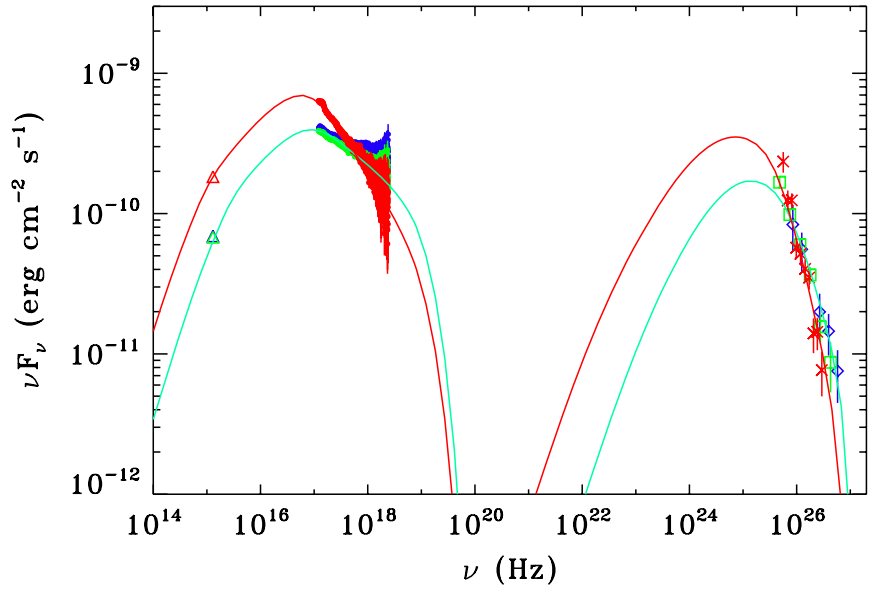

Figure 2. SED with the SSC model for the 2006 (teal blue) and 2008 (red) data. The data from XMM-Newton OM, XMM-Newton EPN, MAGIC, Whipple, and VERITAS are shown with triangles, filled circles, squares, diamonds, and crosses, respectively, with data taken during the MAGIC, Whipple, and VERITAS observation times shown in green, blue and red.

of $3.16 \times 10^{20} \mathrm{~cm}^{-2}, 2.25 \times 10^{20} \mathrm{~cm}^{-2}$, and $4.51 \times 10^{20} \mathrm{~cm}^{-2}$ for the data obtained simultaneously with the MAGIC, Whipple $10 \mathrm{~m}$, and VERITAS observations, respectively. Using these results, the spectrum was unfolded and de-absorbed to derive the intrinsic X-ray spectrum of Markarian 421. Only statistical errors were taken into account here. In addition, the count rates found for the OM exposures were converted to flux using the standard conversion factor ${ }^{58}$ and an average point was determined for each time interval. In addition, using the ultraviolet extinction law from Cardelli et al. (1989), the absolute extinction for the UVM2 band was calculated to be A(UVM2) $=0.13$, allowing for de-reddening of the OM data using a correction factor of 1.13 .

Figure 2 shows the broadband SEDs corresponding to the three epochs of VHE observations. It is important to note that within each epoch the multiwavelength data are genuinely simultaneous. Spectral variability is observed between epochs.

Modeling of the SEDs was carried out using a leptonic model (Böttcher \& Chiang 2002). In this model, the spectral distribution of injected electrons is described by a power law with low- and high-energy cutoffs of $\gamma_{\min }$ and $\gamma_{\max }$, respectively. The emitting region is assumed to be in a state of temporary equilibrium and of spherical shape, with radius $R$, and moves out along the jet at relativistic speed $v / c=\left(1-1 / \Gamma^{2}\right)^{1 / 2}$, where $\Gamma$ is the bulk Lorentz factor. As the emitting region moves along the jet, particles cool due to radiative losses and may escape from the region. The timescale of these escapes is factored into the model as $t_{\mathrm{esc}}=\eta R / c$, with $\eta \geqslant 1$. The radiative processes considered include synchrotron radiation, SSC, and inverse Compton scattering of external photons. However, we found that a model with a negligible contribution from external photons (i.e., a pure SSC model) provides a good match to the SEDs during both observations. In addition, the SED matches have been absorbed with the extragalactic background light model discussed in Franceschini et al. (2008). The values for magnetic field and the spectral index of the injected electrons were varied until a good match was found for the 2006 and 2008 SEDs. The parameters for these models are shown in Table 6 . The models are also shown in Figure 2.

\footnotetext{
58 See http://heasarc.nasa.gov/docs/xmm/sas/USG/node135.html.
} 

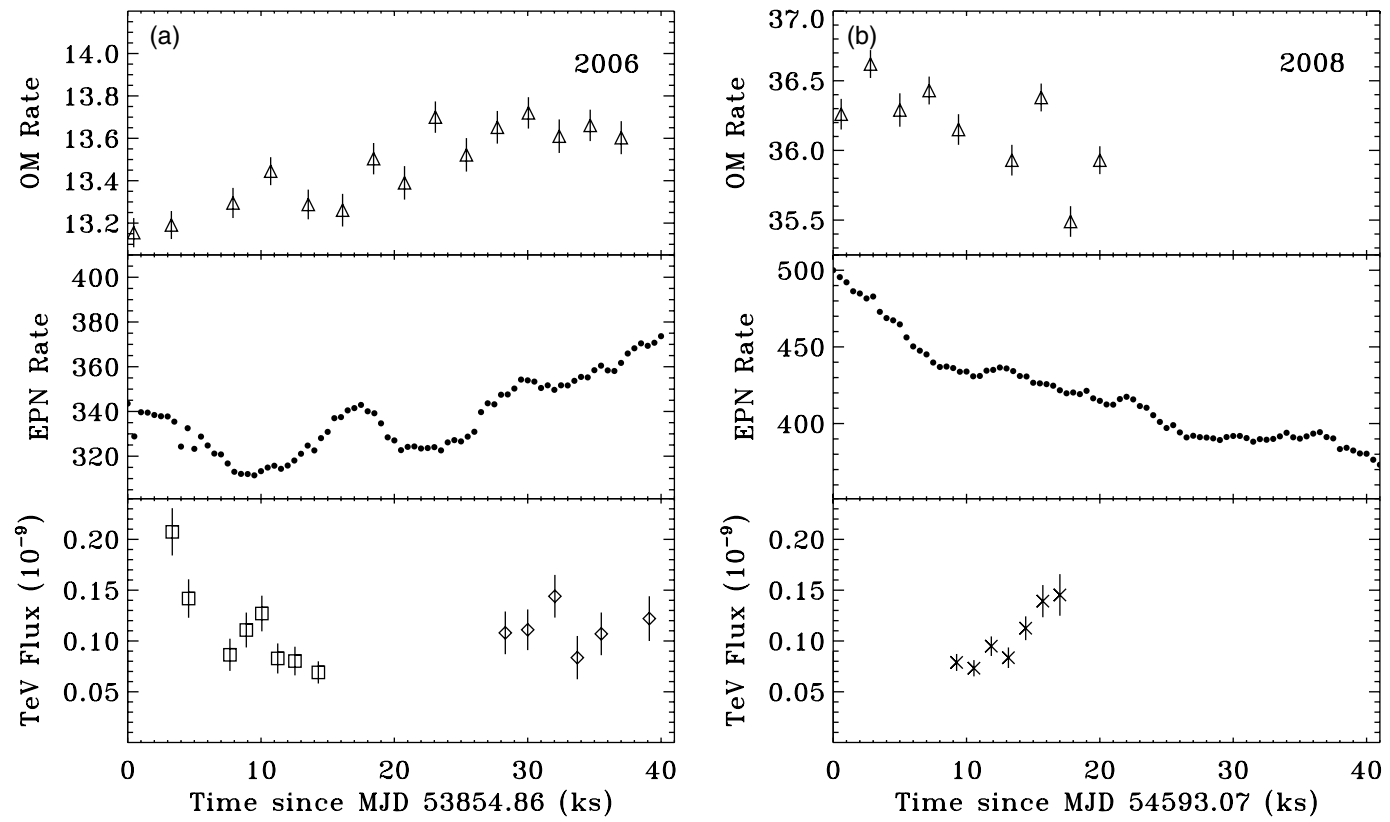

Figure 3. Light curves from (a) 2006 observations and (b) 2008 observations. XMM-Newton OM data (200-300 nm) is shown with triangles in units of counts s ${ }^{-1}$. $X M M$-Newton EPN data $(0.5-10.0 \mathrm{keV})$ is shown with filled circles in units of counts $\mathrm{s}^{-1}$. Error bars for the EPN data are smaller than the data points. The data from MAGIC, Whipple, and VERITAS are shown with squares, diamonds, and crosses, respectively, in units of photons $\mathrm{cm}^{-1} \mathrm{~s}^{-2}$ (above $250 \mathrm{GeV}$ ). Note that the EPN and OM scales differ on the 2006 and 2008 panels.

Table 6

Model Parameters

\begin{tabular}{lcc}
\hline \hline \multicolumn{1}{c}{ Parameter } & 2006 Value & 2008 Value \\
\hline$\gamma_{\min }$ & $4.2 \times 10^{4}$ & $3.3 \times 10^{4}$ \\
$\gamma_{\max }$ & $5.0 \times 10^{5}$ & $4.0 \times 10^{5}$ \\
Injection electron & 2.6 & 3.2 \\
Spectral index & & \\
Escape time parameter & $\eta_{\mathrm{esc}}=3$ & $\eta_{\mathrm{esc}}=3$ \\
$\left(t_{\mathrm{esc}}=\eta R / c\right)$ & & \\
Magnetic field at $z_{0}(\mathrm{G})$ & 0.48 & 0.68 \\
Bulk Lorentz factor & $\Gamma=20$ & $\Gamma=20$ \\
Blob radius $(\mathrm{cm})$ & $3.0 \times 10^{15}$ & $3.0 \times 10^{15}$ \\
Observing angle (deg) & $\theta_{\mathrm{obs}}=2.87$ & $\theta_{\mathrm{obs}}=2.87$ \\
$L_{e}($ jet $)$ & $7.76 \times 10^{42} \mathrm{erg} \mathrm{s}^{-1}$ & $1.06 \times 10^{43} \mathrm{erg} \mathrm{s}^{-1}$ \\
$L_{B}$ (jet) & $3.11 \times 10^{42} \mathrm{erg} \mathrm{s}^{-1}$ & $6.24 \times 10^{42} \mathrm{erg} \mathrm{s}^{-1}$ \\
$L_{B} / L_{e}$ & 0.40 & 0.59 \\
\hline
\end{tabular}

\subsection{Cross-band Correlation}

The VHE, X-ray, and UV light curves are shown in Figure 3 with $1 \sigma$ uncertainties. The X-ray light curve was initially binned using a time interval of $500 \mathrm{~s}$, while the bins for the VHE light curves were primarily determined by the standard length of data runs for the respective telescopes. The Whipple $10 \mathrm{~m}$, MAGIC, and VERITAS data are in 28 minute, 22 minute, and 20 minute bins, respectively. To provide a direct comparison between the results obtained with different VHE experiments, we show the gamma-ray fluxes above a common energy threshold $(250 \mathrm{GeV})$. To reach this threshold for the Whipple $10 \mathrm{~m}$, the flux was extrapolated from the power-law spectrum fit to the data.

In addition, contemporaneous data taken on the Crab Nebula, a standard candle for VHE experiments, were studied to assess systematic uncertainty in the flux calibration of the VHE data. The systematic uncertainty in flux calibration between the MAGIC and VERITAS experiments was estimated to be on the order of $10 \%$ with MAGIC systematically measuring a lower flux than VERITAS. The systematic uncertainty in the Whipple
$10 \mathrm{~m}$ and VERITAS flux calibration was found to be on the order of $40 \%$ with the Whipple $10 \mathrm{~m}$ systematically measuring a higher flux than VERITAS. These systematic uncertainties are not included in the data.

The error bars on the VHE data are relatively large, so in order to quantify the variability, we first tested the entire light curve by fitting a constant flux value to the data. The resulting fit had a $\chi_{v}^{2}$ of 3.92 for 20 degrees of freedom. Similarly, as the MAGIC data made up the most variable portion of the VHE light curve, these data alone were fit to a constant, with a $\chi_{v}^{2}$ of 5.22 for 6 degrees of freedom. This inconsistency of the data with fits to a constant indicates significant variability in the VHE band. Markarian 421 also varied significantly at $\mathrm{X}$-ray energies during the 2006 observation, with the count rate initially decreasing during the course of the MAGIC observation and slowly increasing during the Whipple observation. Though both showed significant variability, the X-ray and VHE data from 2006 do not appear to be correlated.

To examine the X-ray/VHE correlation more closely, we show in Figure 4 the measured VHE flux and X-ray count rates. The X-ray data were rebinned to match the resolution of the corresponding VHE data. To quantify the correlation and examine the uncertainty in this correlation, we used a method similar to that discussed in Albert et al. (2007b). A set of 25,000 light curves was simulated based on the Gaussian errors of the data points in the X-ray and VHE bands, respectively. For each pair of simulated X-ray/VHE light curves, the value of Pearson's $\mathrm{r}$ was calculated. A histogram of these possible $r$ values was generated, giving an average $r$ value of $-0.050 \pm 0.050$, indicating a lack of correlation.

To compare to previous work, the best-fit correlation between the X-ray and VHE bands found in Błażejowski et al. (2005) is plotted in Figure 4 (dotted line). In order to carefully compare this previous result to our results, the PIMMS tool ${ }^{59}$ was used to convert the RXTE count rates to XMM-Newton count

\footnotetext{
59 See http://heasarc.gsfc.nasa.gov/Tools/w3 pimms.html.
} 


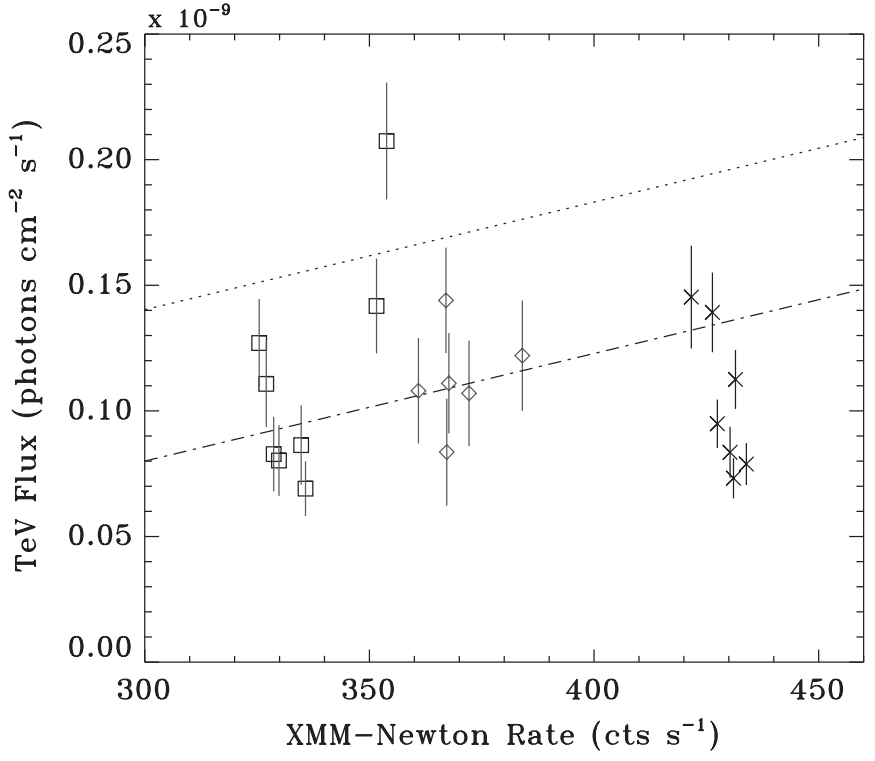

Figure 4. Simultaneous XMM-Newton EPN (0.5-10 keV) and VHE data to search for correlation between the two bands. MAGIC data points are designated by squares, Whipple points by diamonds, and VERITAS points by crosses. All $X M M$-Newton data used for this plot was analyzed with XMMSAS version 8.0.0 to maintain consistency. The dotted line is the $\mathrm{X}$-ray/VHE correlation best fit from Błażejowski et al. (2005) converted to the appropriate units. The dot-dashed line has the same slope but is scaled to the average of our Whipple $10 \mathrm{~m}$ results.

rates, taking into account energy range, spectral shape, and hydrogen column density. To more directly compare our data to Błażejowski et al. (2005), we scaled the correlation to match our Whipple $10 \mathrm{~m}$ points in Figure 4 (dot-dashed line). The overall normalization shift could be a reflection of hysteresis on long timescales. Although the scatter in the VHE points are large, the VERITAS points are systematically below the scaled correlation. This effect is made worse by the fact that the Whipple $10 \mathrm{~m}$ points are known to be systematically higher than the VERITAS points (by 40\%).

There appears to be no correlation between the VHE and UV variations, most notably during the first half of the 2006 observation, where the UV rates increase as the VHE flux decreases. However, the UV rates during both observations appear to roughly follow the trend of the X-ray rates, with a significantly higher rate during the 2008 observation. Using the same method as used above to examine correlations between the VHE and X-ray bands, we found an average value for Pearson's $r$ of $0.940 \pm 0.001$ for the total set of X-ray and UV data, indicating strong correlation.

\subsection{Spectral Hysteresis}

We also examined the spectral evolution of the X-ray data to determine if any hysteresis was present during the observations indicating a dependence of the system on previous states. The data were divided into three energy bands in order to calculate hardness ratios: $0.5-1 \mathrm{keV}, 1-3 \mathrm{keV}$, and 3-10 keV. These bands were chosen such that each band contains a roughly equal number of counts. Figure 5 shows the evolution of the hardness ratios versus intensity (in terms of count rates) through the flare seen in the 2006 data (which peaks near 17-18 ks into the observation; see Figure 3). Spectral hysteresis is clearly seen during this rapid flare. Clockwise patterns indicate a lag in the response of lower-energy photons with respect to that of higherenergy photons. Similar patterns have been seen previously

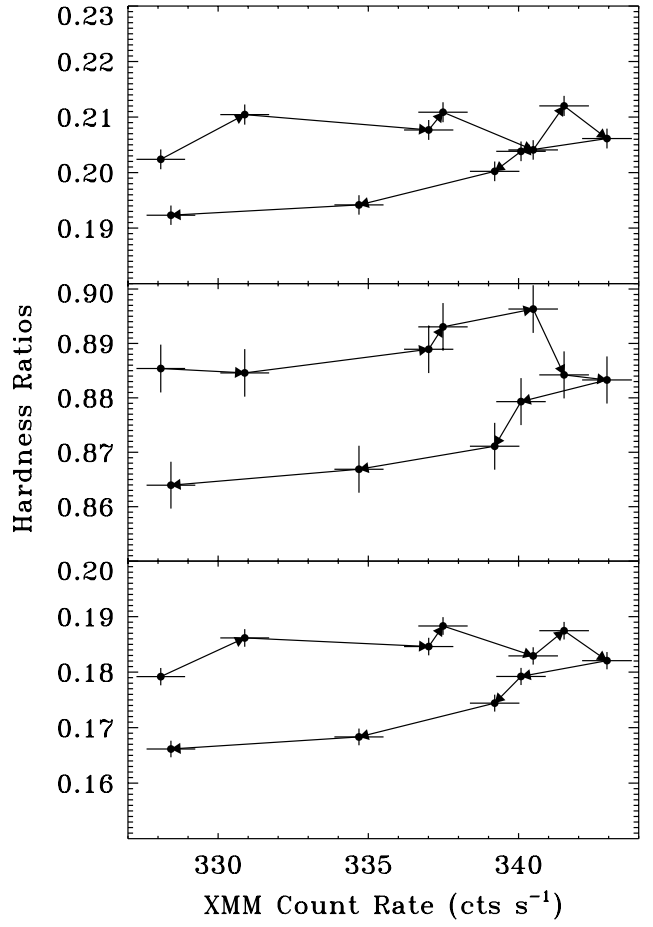

Figure 5. Hardness ratio vs. intensity plots to study spectral hysteresis during the increase in activity centered around $17 \mathrm{ks}$ into the 2006 XMM-Newton observation. Three bands are used for this analysis. The upper panel uses (3$10 \mathrm{keV}) /(1-3 \mathrm{keV})$ for the hardness ratio, the middle panel uses $(1-3 \mathrm{keV}) /$ $(0.5-1 \mathrm{keV})$, and the bottom panel uses $(3-10 \mathrm{keV}) /(0.5-1 \mathrm{keV})$. The arrows indicate the progression of time. Clockwise hysteresis is present in all three panels, indicating a lag in the lower energy bands.

(e.g., Brinkmann et al. 2001), though counter-clockwise patterns have been observed as well (Cui 2004). Different hysteresis patterns indicate a complex mechanism that may differ between outbursts.

No rapid flares were observed during the 2008 observation. Examination of the entire 2008 observation did not reveal any overall spectral hysteresis pattern occurring on the order of several hours. Similarly, no overall hysteresis was found when considering the entire 2006 observation.

\section{DISCUSSION}

This work provides the results from a ToO campaign with the primary focus of studying the rapid flaring activity of blazars on sub-hour timescales in both the X-ray and VHE bands. In addition, these observations expand the pool of truly simultaneous, multiwavelength observations of blazars, essential for the study of correlations during rapid flares. The variability observed during the 2006 outburst in the simultaneous X-ray and VHE observations should be enough to provide useful information about a correlation, if any, between the two energy bands. In addition, the observations from 2008 during a separate outburst provide an extended picture for investigating possible correlations between these two bands. Rapid X-ray and VHE flares are often expected to be correlated in one-zone SSC scenarios, as all photons are expected to originate from the same population of electrons. Examination of the observed SEDs can provide additional constraints on model parameters and reveal what factors strongly affect each flare's spectral profile.

The SED fits show a steeper spectrum for the 2008 observation than for the 2006 observation, particularly at X-ray energies. This is intriguing as previous investigations have observed 
spectral hardening with increased flux (e.g., Xue et al. 2006). In addition, both peaks in the SED show a slight shift to lower energies during the 2008 observation where previous investigation has shown a shift to higher energies with increased luminosity (e.g., Błażejowski et al. 2005). Figure 2 shows that our one-zone SSC model fits both the 2006 and 2008 data quite well.

Historically, multiwavelength monitoring campaigns have observed correlation between the X-ray and VHE emission in Markarian 421, though the data sets are not comprised entirely of strictly simultaneous multiwavelength data (e.g., Błażejowski et al. 2005; Fossati et al. 2008). Though the dynamical range of the observed variability is not as large as that seen in previous long-term monitoring campaigns, we seem to be seeing shortterm variability that shows a different correlation pattern here. Surprisingly, both the 2006 data alone and the entire 2006/ 2008 data set show no obvious correlation, with a Pearson's $r$ for the entire X-ray/VHE data set of $-0.050 \pm 0.050$, though the SSC model fits the SEDs well (see Figures 2 and 4). This implies that the X-ray and VHE photons may originate from electrons with different energies, similar to what was observed during a study of PKS2155-304 (Aharonian et al. 2009). Other scenarios that could explain the observed variability patterns include the possibility of an inhomogeneous emission region or hadronic origin of the VHE emission. It is interesting to note that Markarian 421 is clearly behaving very differently here from what is usually reported of the source (e.g., Błażejowski et al. 2005), where the X-ray and VHE variabilities are seen to be strongly correlated. Such personality makes it difficult to generalize the results to other blazars.

In addition, the OM allowed for a detailed search for UV/Xray or UV/VHE correlations. Though previous multiwavelength campaigns on Markarian 421 have obtained optical, X-ray, and VHE data, correlations between the X-ray and optical data have either not been studied or were not significant (e.g., Albert et al. 2007a; Błażejowski et al. 2005; Horan et al. 2009). This study found the average rate from the XMM-Newton PN detector during the 2008 observation was about $20 \%$ higher than the rates measured during the 2006 observation. Examination of the OM data in the UV band shows that the count rate more than doubles between the 2006 and 2008 observation. The UV rate also appears to vary in step with the X-ray rate within the individual observations. Calculation of the Pearson's $r$ value for the total $\mathrm{UV} / \mathrm{X}$-ray data set yielded a value of $0.940 \pm 0.004$, indicating a strong correlation. This provides direct observational evidence for a link between the emission mechanisms at X-ray and UV wavelengths. On the other hand, we found no apparent UV/ VHE or X-ray/VHE correlation, implying that the seed photons for the inverse Compton process (to produce the VHE photons) cannot be provided by the UV or X-ray emission observed.

We also searched for hysteresis patterns in the X-ray data to provide further information about the outbursts. Hysteresis was observed during one rapid flare in the X-ray data from the 2006 observation. No rapid flares occurred during the 2008 observation. Spectral hysteresis has been commonly observed in blazars, but the phenomenon is not yet understood completely. In Kirk \& Mastichiadis (1999), a simple model is used to produce spectral hysteresis patterns that may be observed during the course of a flare. In this model, the behavior is characterized by the relationship between three timescales associated with the duration of the flare variability $\left(t_{\mathrm{var}}\right)$, synchrotron cooling $\left(t_{\text {cool }}\right)$, and particle acceleration $\left(t_{\text {acc }}\right)$. The relationship between these timescales result in four possible cases that are discussed in detail. The clockwise hysteresis found in the X-ray data, indicating a lag at lower energies in the X-ray band, coupled with the essentially symmetric shape of the flare in the X-ray light curve seems to indicate that the case with $t_{\text {cool }} \gg t_{\mathrm{var}} \gg t_{\mathrm{acc}}$ is most relevant to this observation. The lag in the low-energy photons is a result of the inverse relationship between $t_{\text {cool }}$ and the energy of the cooling particles. Although we observed clockwise patters in one of the observations, other patterns have also been observed. This is yet another example of the personality of the source and may indicate physical differences between individual flares and outbursts.

The VERITAS research was supported by grants from the U.S. Department of Energy, the U.S. National Science Foundation and the Smithsonian Institution, by NSERC in Canada, by Science Foundation Ireland, and by STFC in the U.K. We acknowledge the excellent work of the technical support staff at the FLWO and the collaborating institutions in the construction and operation of the instrument. D.G. and W.C. wish to acknowledge support by NASA through grants NNX06AB96G, NNX08AD76G, and NNX08AX53G. The MAGIC Collaboration thanks the Instituto de Astrofisica de Canarias for the excellent working conditions at the Observatorio del Roque de los Muchachos in La Palma. The support of the German BMBF and MPG, the Italian INFN, and Spanish MCINN is gratefully acknowledged. This work was also supported by ETH Research Grant TH 34/043, by the Polish MNiSzW Grant N N203 390834, and by the YIP of the Helmholtz Gemeinschaft.

Facilities: MAGIC, VERITAS, FLWO:10m, XMM

\section{REFERENCES}

Aharonian, F. A. 2000, New Astron., 5, 377

Aharonian, F. A., et al. 2009, ApJ, 696, L150

Albert, J., et al. (MAGIC Collaboration) 2007a, ApJ, 663, 125

Albert, J., et al. (MAGIC Collaboration) 2007b, ApJ, 669, 862

Albert, J., et al. (MAGIC Collaboration) 2007c, Nucl. Instrum. Methods Phys. Res. A, 583, 494

Albert, J., et al. (MAGIC Collaboration) 2008, ApJ, 674, 1037

Albert, J., et al. (MAGIC Collaboration) 2009, A\&A, 493, 467

Anderhub, H., et al. (MAGIC Collaboration) 2009, A\&A, 498, 83

Baixeras, C., et al. (MAGIC Collaboration) 2004, Nucl. Instrum. Method, A588, 494

Berge, D., Funk, S., \& Hinton, J. 2007, A\&A, 466, 1219

Błażejowski, M., et al. 2005, ApJ, 630, 130

Böttcher, M., \& Chiang, J. 2002, ApJ, 581, 127

Breiman, L. 2001, Mach. Learn., 45, 5

Bretz, T. (MAGIC Collaboration) 2005, Proc. 29th ICRC (Pune), 4, 315

Bretz, T., \& Dorner, D. 2008, AIP Conf. Proc., 1085, 664

Brinkmann, W., et al. 2001, A\&A, 365, L162

Cardelli, J. A., et al. 1989, ApJ, 345, 245

Cogan, P., et al. 2007, arXiv:0709.4233

Cortina, J., et al. (MAGIC Collaboration) 2005, Proc. 29th ICRC (Pune), 5, 359

Cui, W. 2004, ApJ, 605, 662

Daum, A., et al. 1997, Astropart. Phys., 8,

Dermer, C. D., Schlickeiser, R., \& Mastichiadis, A. 1992, A\&A, 256, L27

Fomin, V. P., et al. 1994, Astropart. Phys., 2, 137

Fossati, G., et al. 2008, ApJ, 677, 906

Franceschini, A., et al. 2008, A\&A, 487, 837F

Gabriel, C., et al. 2004, in ASP Conf. Ser. 314, Astronomical Data Analysis Software and Systems (ADASS) XIII, ed. F. Ochsenbein, M. G. Allen, \&

D. Egret (San Francisco, CA: ASP) 759

Gaidos, J. A., et al. 1996, Nature, 383, 319

Gaug, M., Bartko, H., Cortina, J., \& Rico, J. (MAGIC Collaboration) 2005, Proc. 29th ICRC (Pune), 5, 375

Hillas, A. M. 1985, Proc. 19th ICRC (La Jolla), 3, 445

Horan, D., et al. 2009, ApJ, 695, 596

Kildea, J., et al. 2007, Astropart. Phys., 28, 182

Kirk, J. G., \& Mastichiadis, A. 1999, Astropart. Phys., 11, 45 
Konopelko, A. 1995, in Proc. Padova Workshop on TeV Gamma-Ray Astrophysics, Towards a Major Atmospheric Cerenkov Detector IV, ed. M. Cresti (Piazzola sul Brenta: Papergraf), 373

Mannheim, K. 1993, A\&A, 269, 67

Maraschi, L., et al. 1992, ApJ, 397, L5

Marscher, A. P., \& Gear, W. K. 1985, ApJ, 298, 114

Mason, K. O., et al. 2001, A\&A, 365, L36

Mohanty, G., et al. 1998, Astropart. Phys., 9, 15
Pohl, M., \& Schlickeiser, R. 2000, A\&A, 354, 395

Punch, M., et al. 1991, Proc. 22nd ICRC (Dublin), 1, 464

Punch, M., et al. 1992, Nature, 358, 477

Riegel, B., et al. (MAGIC Collaboration) 2005, Proc. 29th ICRC (Pune), 5, 215

Sikora, M., Begelman, M. C., \& Rees, M. J. 1994, ApJ, 421, 153

Strüder, L., et al. 2001, A\&A, 365, L18

Urry, C. M., \& Padovani, P. 1995, PASP, 107, 803

Xue, Y., Yuan, F., \& Cui, W. 2006, ApJ, 647, 194 